\title{
Effectiveness of personal letters to healthcare professionals in changing clinical practice behaviours: A Systematic Review protocol
}

Aikaterini Grimani ( $\square$ aikaterini.grimani@wbs.ac.uk)

University of Warwick

Louis Goffe

Newcastle University

Mei Yee Tang

Newcastle University

Fiona Beyer

Newcastle University

\section{Falko F. Sniehotta}

Newcastle University

Ivo Vlaev

University of Warwick

\section{Protocol}

Keywords: healthcare professionals, personal letters, behavioural change, systematic review

Posted Date: August 13th, 2020

DOl: https://doi.org/10.21203/rs.3.rs-55534/v1

License: () (1) This work is licensed under a Creative Commons Attribution 4.0 International License.

Read Full License

Version of Record: A version of this preprint was published at Systematic Reviews on April 2nd, 2021. See the published version at https://doi.org/10.1186/s13643-021-01650-4. 


\section{Abstract}

Background: Letters are regularly sent by healthcare organisations to healthcare professionals to encourage them to take action, change practice or implement guidance. However, whether letters are an effective tool in delivering a change in clinical practice behaviour is currently uncertain. In addition, there are currently no evidence-based guidelines to support providers and health authorities with advice on how to formulate the communication, what information and behaviour change techniques to include in order to optimise the potential effect on the behaviour of the receivers. To address this research gap, we aim to inform such guidance through this systematic review.

Methods/ Design: A systematic literature search of published and unpublished studies (the grey literature) in electronic databases will be conducted to identify studies that meet our inclusion criteria. The search will be conducted in five electronic databases: MEDLINE, EMBASE, PsycINFO, the Cochrane Library and CINAHL. We will also conduct supplementary searches in Google Scholar, hand search relevant journals, and conduct backward and forward citation searching for included studies and relevant reviews. A systematic approach to searching, screening, reviewing and data extraction will be applied based on Preferred Reporting Items for Systematic reviews and Meta-Analysis. Titles, abstracts, full-texts for eligibility will be examined independently by researchers. The quality of the included studies will be assessed using quality assessment tool for studies with diverse design and Cochrane risk of bias tool. Disagreements will be resolved by a consensus procedure.

Discussion: This review aims to provide comprehensive evidence of the effectiveness of personal letters to healthcare professionals in changing clinical practice behaviours. Health policy makers across government will benefit from being able to increase compliance in clinical settings by applying theories of behaviour to design of policy communications. The synthesized findings will be disseminated through peer-reviewed publication.

\section{Systematic review registration: PROSPERO CRD42020167674}

\section{Background}

Letters are regularly sent by healthcare organisations to healthcare professionals (HCPs) to encourage them to take action, change practice or implement guidance (e.g. reduction in antibiotic prescribing (1), raise performance of mental health clinicians (2), reduction in sedative hypnotic medications prescribing (3)). Letters are inexpensive, usually personalised and have wide reach (1). Common definitions define letters as "a written, typed, or printed communication, sent in an envelope by post or messenger" (4), "a written message from one person to another, usually put in an envelope and sent by post" (5), "a direct or personal written or printed message addressed to a person or organization" (6). In addition, emails are usually defined as "written messages distributed by electronic means via a network" (4).

Whether letters are an effective tool in delivering a change in clinical practice behaviour is currently uncertain, though. The delivery of an intervention can be described through the use of behaviour change 
techniques which can be helpful when trying to examine to what extent the barriers and facilitators to a behaviour are addressed in interventions. BCTs are the discrete and definable components of the given interventions that on their own have the potential to change behaviour. They are observable and replicable and can be used alone or in combination with other BCTs (7). A BCT is an "active ingredient" such as self-monitoring of behaviour (e.g., keeping a daily diary of food consumption) (8). Effective BCTs have been identified for interventions to increase physical activity and healthy eating and to support smoking cessation, safe drinking, prevention of sexually transmitted infections, and changing professional behaviour (7). However, there is currently no evidence about behaviour change techniques (BCTs) (7) used in letters. Other approaches such as the MINDSPACE framework (9) may optimise effects of letters. The MINDSPACE framework, consists of nine evidence based principles of behaviour change messenger, incentives, norms, defaults, salience, priming, affect, commitment and ego- is becoming widely used within the policymaking community to improve the effectiveness of existing and new behaviour change policies $(9,10)$. Likewise, there are a range of approaches to enhance behaviour change responses to letters, such as adding additional materials (e.g., a leaflet) or follow-up phone calls or text messages which might help boosting effects (1).

Letters are promising in delivering behaviour change and that the utilisation of particular BCTs boost the effects. For example, Hallsworth (1) found that a letter containing a social norm message sent to General Practitioners (GPs) to notify them that their practice is prescribing antibiotics at a higher rate than $80 \%$ of the practices in their local area reduced the prescribing rate compared to GPs who did not receive a letter. This study also revealed that a message from a high-profile sender (known as messenger effect) substantially reduced antibiotic prescribing at low cost and at national scale.

Despite the impact shown by selective studies for letters to be an effective tool in changing clinical practice behaviours, to date, there has not been a comprehensive review to assess their potential more broadly in the role of population health. To address this research gap, we will conduct a systematic review that will identify what information and behaviour change techniques within letters that have the potential to optimise the effect on the behaviour of healthcare professionals.

\section{Objective}

The aim of this research is to conduct a systematic review of Randomised Controlled Trials (RCTs) evaluating interventions that include letters sent to HCPs to alter their professional clinical behaviour and to identify lessons for improved letter design leading to behavioural compliance.

Research questions:

- Are personal letters sent from healthcare organisations effective in changing the clinical behaviour of HCPs?

- What are the BCTs within letters sent to HCP that have been evaluated?

- What BCTs are particularly effective in changing healthcare professional behaviour? 
- Does the effectiveness of letters sent to change healthcare professional behaviour vary by:

1. Features of the sender

2. Features of the receiver

3. Features of the target behaviour (e.g., prescription, referral, diagnostic test, etc.)

4. Mode of message email vs. letter

5. Additional content (e.g., added leaflet)

6. Additional communications (e.g. follow-up calls, tests, reminder letters)

7. Other letter features that may not be classified according to the existing taxonomies (e.g. adding signature) $(7,9)$

\section{Methods/ Design}

The systematic review will be performed following the Preferred Reporting Items for Systematic Reviews and Meta-Analyses (PRISMA) statement $(11,12)$. The protocol was registered in the International Prospective Register of Systematic Reviews (number CRD42020167674) (13).

A comprehensive literature search of published and unpublished studies ("grey literature") in electronic databases will be conducted. For unpublished studies we will contact partners (e.g. UK government departments, agencies and public bodies such as Public Health England; the Behavioural Insights Team). The search will be conducted in five electronic databases: Medline, EMBASE.com, PsycINFO, Cochrane Library and CINAHL. We will conduct supplementary searches in Google Scholar, hand search relevant journals, and conduct backward and forward citation searching of included studies and relevant reviews.

A search strategy (Additional file 1) following PICOS will be adapted (population, intervention, comparison, outcome and study design), including Medical Subject Headings (MeSH) terms and relevant key words $(14,15)$. The PICOS process facilitates a more evidence-based approach to literature searching and helps to rapidly and accurately locate the best available scientific information, avoiding unnecessary searching (15).

\section{Eligibility criteria}

All studies written in English language published until March 2020 will be considered. The specific inclusion criteria of the study will be the following:

- Types of studies to be included: We will include published and unpublished (grey literature) RCTs, which excludes non-randomized study designs with a predefined control group or comparator group (excluding also before-and-after studies and 'natural experiments'). Due to our close relationships with the Department of Health and Social Care (DHSC) and behavioural science units across Europe as a Policy Research Unit, we are in a strong position to identify additional grey literature not (yet) published in peer reviewed journals. 
- Population: We will include HCPs who are involved in providing direct patient care (this includes HCPs in training). No restrictions were placed on geographical region.

- Interventions: We will be looking for interventions focused on letters sent from healthcare organisations to HCPs with the intent to alter their professional practice. We will also consider emails, but classifying them separately in our analysis (including interventions in which the letter is the content of an email or an attachment to an email).

- Comparisons: Relevant comparisons will include no intervention control arms, non-personalised information such as circulars or newsletters, letters with different format or content or letters with additional materials (such as leaflets) or reminders (by phone, text or letter).

- Primary Outcomes: Measures of health professional clinical behaviour such as rates of performing prevention, diagnosis, and treatment behaviours (e.g. immunisation, blood pressure measurement, prescription, referral); measures of health professional non-clinical behaviour such as rates of performing specified non-clinical behaviours (for example achieve recruitment targets for clinical trials). No secondary outcomes will be included.

- Study designs: We will focus on RCT designs.

\section{Patient and public involvement (PPI)}

PPI involvement has the ability to empower people with respect to health and social care services, influencing change and improvement upon those people the services most concern (16). A core component of PRU (Policy Research Unit) policy is to maximise public involvement in all our research activities (17). Thus, the PRU PPI panel has commented on the protocol and will receive regular updates on the review and comment on outputs.

\section{Selection of studies}

All titles and abstracts retrieved by electronic searching will be downloaded to the reference management database End Note and uploaded to Covidence, one of Cochrane's recommended tools. This web-based software platform has been designed to support more efficient management of systematic reviews and can be used from the beginning of title/abstract screening through the beginning of meta-analysis (18). Duplicates will be removed and two review authors independently will examine the remaining references. The abstracts will be included if they meet the inclusion criteria. Subsequently, two reviewers will independently determine the eligibility of studies on the basis of a review of the full texts. Differences in judgment will be resolved through discussion and inclusion of a third rater. The selection process will be recorded and the Preferred Reporting Items for Systematic Reviews and Meta-Analyses flow diagram will be completed (11).

\section{Data extraction}

The process of data extraction will involve one or two reviewers who generate a data extraction form. The data extraction form, which will be reviewed and refined by the reviewers, will include variables as 
follows:

- characteristics of the receiver

- characteristics of the sender

- intervention features including

- Template for Intervention Description and Replication (TIDieR) checklist: (brief name, why, what (materials), what (procedure), who provided, how, where, when and how much, tailoring, modifications, how well (planned), how well (actual) (19).

- MINDSPACE checklist of behavioural economic techniques: the nine most robust effects on behaviour (Messenger, Incentives, Norms, Defaults, Salience, Priming, Affect, Commitment and Ego) $(9,20)$.

- BCTs (observable and replicable components designed to change behaviour) $(7,21)$, intervention functions (education, persuasion, incentivisation, coercion, training, restriction, environmental restructuring, modelling, enablement) and policy categories (communication/ marketing, guidelines, fiscal, regulation, legislation, environmental/ social planning, service provision), which are all part of the Behaviour Change Wheel framework $(7,22)$.

- Primary outcome(s): Measures of health professional clinical and non-clinical behaviour. Where more than one reported outcome is provided, we will use the Grades of Recommendation, Assessment, Development and Evaluation (GRADE) approach to assess the certainty of evidence (separated into those that are critical, important and not important) (23). Outcomes measured at multiple time points will be categorized as following: immediate (within 2 weeks of the intervention delivery), short-term (2-13 weeks after intervention delivery), medium-term (1450 weeks after intervention delivery) and long-term effects (51 or more weeks after intervention delivery). We will present multiple time points only for critical outcomes.

\section{Quality assessment}

An assessment of the methodological quality of included studies will be conducted using the Cochrane Collaboration Risk of Bias Tool (CCRBT) (24). CCRBT is a two-part tool, addressing seven evidence-based domains, namely random sequence generation (selection bias), allocation concealment (selection bias), blinding of participants and personnel (performance bias), blinding of outcome assessment (detection bias), incomplete outcome data (attrition bias), selective outcome reporting (reporting bias) and other sources of bias (other bias). The first part of the tool describes a sufficiently detailed support for judgment about the risk of bias, ensuring its transparency. The second part assigns a judgment relating to the risk of bias for each domain. This is achieved by assigning a judgment of 'Low risk' of bias (+), 'High risk' of bias (-), or 'Unclear risk' of bias (?). According to the Cochrane Collaboration's recommendations, the studies, whose all domains will be rated positively, will be judged as low risk of bias, while the studies with one or more unclear domains will be judged as having unclear risk of bias. Furthermore, the studies, where one or more domains will be rated negatively, will be judged as high risk of bias $(25,26)$. Two review authors will independently evaluate the methodological quality of each 
included study using the assessment tools. Discrepancies will be resolved through a consensus procedure.

\section{Data analysis and synthesis}

We will compare the reported intervention features against a control condition which does not contain those features. This will provide information useful for explaining why interventions were effective or ineffective. The analyses of those comparisons will be conducted and reported separately according to the participants' characteristics and characteristics of the sender.

BCTs will be double-coded by trained coders using the Behaviour Change Techniques Taxonomy v1 (7) using a BCT extraction form. BCTs will be coded separately for intervention and control groups. The reliability of coding of BCTs, MINDSPACE checklist, and TiDIER checklist (namely the presence or absence of codes within each intervention) will be assessed using the prevalence and bias-adjusted kappa (PABAK) statistic (27). PABAK was used because adjusts for shared bias in the coders' use of categories and high prevalence of negative agreement (i.e. when both coders agree that codes are absent). A random-effects meta-analysis with the extracted BCTs will be conducted, if the data permits. The meta-analysis will be conducted when a group of studies will be sufficiently homogeneous in terms of subjects involved, interventions, and outcomes to provide a meaningful summary. Although a metaanalysis can be conducted with minimum 2 studies, Valentine, Pigott (28) suggest that the combination of very few studies with heterogeneous characteristics makes any kind of synthesis untenable in most cases, while parameter estimation (e.g., the random effects variance component) will likely be poor, rendering conclusions that are highly uncertain.

\section{Results}

\section{Quality assessment}

An assessment of the methodological quality of included studies will be conducted using the Cochrane Collaboration Risk of Bias Tool (CCRBT) (24). CCRBT is a two-part tool, addressing seven evidence-based domains, namely random sequence generation (selection bias), allocation concealment (selection bias), blinding of participants and personnel (performance bias), blinding of outcome assessment (detection bias), incomplete outcome data (attrition bias), selective outcome reporting (reporting bias) and other sources of bias (other bias). The first part of the tool describes a sufficiently detailed support for judgment about the risk of bias, ensuring its transparency. The second part assigns a judgment relating to the risk of bias for each domain. This is achieved by assigning a judgment of 'Low risk' of bias (+), 'High risk' of bias (-), or 'Unclear risk' of bias (?). According to the Cochrane Collaboration's recommendations, the studies, whose all domains will be rated positively, will be judged as low risk of bias, while the studies with one or more unclear domains will be judged as having unclear risk of bias. Furthermore, the studies, where one or more domains will be rated negatively, will be judged as high risk of bias $(25,26)$. Two review authors will independently evaluate the methodological quality of each 
included study using the assessment tools. Discrepancies will be resolved through a consensus procedure.

\section{Data analysis and synthesis}

We will compare the reported intervention features against a control condition which does not contain those features. This will provide information useful for explaining why interventions were effective or ineffective. The analyses of those comparisons will be conducted and reported separately according to the participants' characteristics and characteristics of the sender.

BCTs will be double-coded by trained coders using the Behaviour Change Techniques Taxonomy v1 (7) using a BCT extraction form. BCTs will be coded separately for intervention and control groups. The reliability of coding of BCTs, MINDSPACE checklist, and TiDIER checklist (namely the presence or absence of codes within each intervention) will be assessed using the prevalence and bias-adjusted kappa (PABAK) statistic (27). PABAK was used because adjusts for shared bias in the coders' use of categories and high prevalence of negative agreement (i.e. when both coders agree that codes are absent). A random-effects meta-analysis with the extracted BCTs will be conducted, if the data permits. The meta-analysis will be conducted when a group of studies will be sufficiently homogeneous in terms of subjects involved, interventions, and outcomes to provide a meaningful summary. Although a metaanalysis can be conducted with minimum 2 studies, Valentine, Pigott (28) suggest that the combination of very few studies with heterogeneous characteristics makes any kind of synthesis untenable in most cases, while parameter estimation (e.g., the random effects variance component) will likely be poor, rendering conclusions that are highly uncertain.

\section{Discussion}

This knowledge synthesis can serve as a guide for effective interventions to support providers and health authorities with advice on how to formulate the communication, what information and BCTs to include in order to optimise the potential effect on the behaviour of the receivers (HCPs). Therefore, we will develop a guide to help staff optimise communications with HCPs. Reaching people with a message is one thing, influencing and changing their behaviour is quite another. Our behavioural science review will provide tried and tested methods that help us design communications that more effectively influence the decisions HCPs make. This guide will include a checklist that details step by step instructions on how to design, develop and test behaviourally informed communications. It will include, tips, techniques and examples of how the methods outlined have been used successfully around the globe in recent years. We will try to keep the guide as simple and accessible as possible. It should help to quickly learn about the theory, steps and techniques that can be applied to design more effective communications. Whether we want our recipients to start, stop or change a behaviour, following the checklist will help influencers to get the response they want. In particular, the review will inform the development of the multi-step checklist which will include sections such as Receiver, Action, Sender, Channel, Techniques. In particular: 
- Receiver: Who is the target audience? This step includes also finding opportunities for segmenting your target audience - i.e. grouping HCPs together by tendencies and characteristics so one can tailor the message to suit the characteristics of each different group.

- Action: What is the desired response? This step involves clearly defining the behavioural response required by the recipient(s) of the communication.

- Sender: Who is the sender of the letter? The receiver may respond differently depending on who is the messenger (e.g. central government, local authority, senior manager, regulator, clinical/professional body, nongovernmental organization, private company, etc.).

- Channel: What is the best mode of letter delivery (e.g. post, email, sms, in person) to maximise engagement for the selected Recipient and Effect?

- Techniques: Which BCTs can be used to design the communications for the selected Recipient, Effect, and Channel? Whilst techniques come in many different forms, we will list the ones that have proven to be particularly effective and useful when designing communications. For example, people are more likely to respond when they feel they are being addressed as individuals and not just a 'number'. Also, does the letter grab and hold the reader's attention? Is the language simple, clear and easy to understand? Is there a clear call to action (i.e. can the reader understand what is being asked of them and by when within the first few lines of the document)? Does it use the right tone of voice?

\section{Strengths and limitations of this study}

The behavioural analysis, which will be carried out using the Behaviour Change Wheel framework, will identify the key behaviours and, importantly, drivers for behaviours that may be amenable to change. The review will inform the development of a multi-step checklist which will include sections such as Receiver, Action, Sender, Channel, Techniques, giving important insights and practical applications for policymakers, health insurers, and health systems. The inclusion of grey literature will broaden this study in terms of included information. Limitations of this review include the exclusion of papers reported in languages other than English.

\section{Reaching conclusions}

We will base our conclusions on findings from the narrative synthesis of included studies for this review. Health policy makers across government will benefit from being able to increase compliance in clinical settings by applying theories of behaviour to design of policy communications. In particular, letters are continuously used by health systems to influence HCPs.

There is growing evidence that BCTs can positively influence behaviours in a range of clinical situations. However, it remains unclear what BCTs have been most effective and how physicians experienced intervention letters (e.g. as helpful feedback or as a scare tactic). Ultimately, this kind of multicomponent intervention may have been the most effective approach considering that the low cost of the letter means that even a relatively small effect size could be cost-effective. These are important insights for 
policymakers, health insurers, and health systems as they jointly pursue efforts to improve the value of care.

\section{Abbreviations}

HCPs: Healthcare Professionals; BCTs:Behavioural Change Techniques; GPs:General Practitioners; PPI:Patient and public involvement; TIDieR:Template for Intervention Description and Replication; GRADE:Grades of Recommendation, Assessment, Development and Evaluation; PABAK:prevalence and bias-adjusted kappa; PRISMA:Preferred Reporting Items for Systematic Reviews and Meta-Analyses; Embase:Excerpta Medica Database; MEDLINE:Medical Literature Analysis and Retrieval System Online; CINAHL:Cumulative Index to Nursing and Allied Health Literature; PICOS:Population, Intervention, Comparison, Outcome, Study design; RCT:Randomized Control Trial; MeSH:Medical Subject Headings; CCRBT:Cochrane Collaboration Risk of Bias Tool

\section{Declarations}

Ethics approval and consent to participate: Not applicable.

Consent for publication: Not applicable.

Availability of data and materials: Not applicable.

Competing interests: The authors declare that they have no competing interests.

Funding statement: This study/project is funded by the National Institute for Health Research (NIHR) [Policy Research Unit in Behavioural Science (project reference PR-PRU-1217-20501)]. The views expressed are those of the author(s) and not necessarily those of the NIHR or the Department of Health and Social Care.

Author Contributions: AG is the corresponding author and the primary author of the study, contributed to the development of the search strategy, developed the inclusion and exclusion criteria and data extraction criteria. LG contributed to the development of the search strategy and provided written feedback on the manuscript. MYT contributed to the development of the search strategy and provided written feedback on the manuscript. FFS conceived the study, contributed to the development of the selection criteria and data extraction criteria and provided written feedback on the manuscript. FB contributed to the development of the search strategy. IV conceived the study, contributed to the development of the selection criteria and data extraction criteria, was involved in the conceptualization of the research question and revised the manuscript critically and contributed to it intellectually. All authors have read and approved the final version of the manuscript.

Acknowledgments: Not applicable

\section{References}


1. Hallsworth M. Increasing compliance in policy settings by applying psychological theories of behaviour to message design. 2017.

2. Azocar F, Cuffel B, Goldman W, McCarter L. The impact of evidence-based guideline dissemination for the assessment and treatment of major depression in a managed behavioral health care organization. The journal of behavioral health services research. 2003;30(1):109-18.

3. Smith DH, Christensen DB, Stergachis A, Holmes G. A randomized controlled trial of a drug use review intervention for sedative hypnotic medications. Medical care. 1998:1013-21.

4. Stevenson A. Oxford dictionary of English. USA: Oxford University Press; 2010.

5. McCreary DR. Cambridge Academic Content Dictionary. Dictionaries: Journal of the Dictionary Society of North America. 2009;30(1):151-5.

6. Staff M-W. Merriam-Webster's collegiate dictionary: Merriam-Webster; 2004.

7. Michie S, Richardson M, Johnston M, Abraham C, Francis J, Hardeman W, et al. The behavior change technique taxonomy $(\mathrm{v} 1)$ of 93 hierarchically clustered techniques: building an international consensus for the reporting of behavior change interventions. Annals of behavioral medicine. 2013;46(1):81-95.

8. Cane J, Richardson M, Johnston M, Ladha R, Michie S. From lists of behaviour change techniques (BCT s) to structured hierarchies: comparison of two methods of developing a hierarchy of BCT s. $\mathrm{Br}$ J Health Psychol. 2015;20(1):130-50.

9. Dolan P, Hallsworth M, Halpern D, King D, Metcalfe R, Vlaev I. Influencing behaviour: The mindspace way. J Econ Psychol. 2012;33(1):264-77.

10. Yoong SL, Hall A, Stacey F, Grady A, Sutherland R, Wyse R, et al. Nudge strategies to improve healthcare providers' implementation of evidence-based guidelines, policies and practices: a systematic review of trials included within Cochrane systematic reviews. Implementation Science. 2020;15(1):50.

11. Moher D, Shamseer L, Clarke M, Ghersi D, Liberati A, Petticrew M, et al. Preferred reporting items for systematic review and meta-analysis protocols (PRISMA-P) 2015 statement. Systematic reviews. 2015;4(1):1.

12. Shamseer L, Moher D, Clarke M, Ghersi D, Liberati A, Petticrew M, et al. Preferred reporting items for systematic review and meta-analysis protocols (PRISMA-P) 2015: elaboration and explanation. Bmj. 2015;349.

13. Grimani A, Goffe L, Tang MY, Sniehotta F, Vlaev I. The effectiveness of personal letters to healthcare professionals in changing clinical practice behaviours: a systematic review: PROSPERO 2020 CRD42020167674; [Available from: https://www.crd.york.ac.uk/prospero/display_record.php? ID=CRD42020167674.

14. Huang M, Névéol A, Lu Z. Recommending MeSH terms for annotating biomedical articles. J Am Med Inform Assoc. 2011;18(5):660-7.

15. Methley AM, Campbell S, Chew-Graham C, McNally R, Cheraghi-Sohi S. PICO, PICOS and SPIDER: a comparison study of specificity and sensitivity in three search tools for qualitative systematic 
reviews. BMC Health Serv Res. 2014;14(1):579.

16. Hayes H, Buckland S, Tarpey M. Briefing notes for researchers: public involvement in NHS, public health and social care research. INVOLVE: Eastleigh; 2012.

17. PRU Behavioural Science. Partnership Working: Patient and Public Engagement 2020 [Available from: https://research.ncl.ac.uk/behscipru/partnership\%20working/.

18. Covidence. Cochrane Community; [Available from: https://community.cochrane.org/help/tools-andsoftware/covidence.

19. Hoffmann TC, Glasziou PP, Boutron I, Milne R, Perera R, Moher D, et al. Better reporting of interventions: template for intervention description and replication (TIDieR) checklist and guide. Bmj. $2014 ; 348$.

20. Vlaev I, King D, Dolan P, Darzi A. The theory and practice of "nudging": changing health behaviors. Public Administration Review. 2016;76(4):550-61.

21. Michie S, Wood CE, Johnston M, Abraham C, Francis J, Hardeman W. Behaviour change techniques: the development and evaluation of a taxonomic method for reporting and describing behaviour change interventions (a suite of five studies involving consensus methods, randomised controlled trials and analysis of qualitative data). Health Technology Assessment. 2015;19(99).

22. Michie S, Van Stralen MM, West R. The behaviour change wheel: a new method for characterising and designing behaviour change interventions. Implementation science. 2011;6(1):42.

23. Schünemann HJ, Higgins JP, Vist GE, Glasziou P, Akl EA, Skoetz N, et al Completing 'Summary of findings' tables and grading the certainty of the evidence. In: Higgins JPT, Thomas J, Chandler J, Cumpston M, Li T, Page MJ, et al., editors. Cochrane Handbook for Systematic Reviews of Interventions: The Cochrane Collaboration; 2019. p. 375-402.

24. Higgins JP, Altman DG, Gøtzsche PC, Jüni P, Moher D, Oxman AD, et al. The Cochrane Collaboration's tool for assessing risk of bias in randomised trials. Bmj. 2011;343:d5928.

25. Higgins JP, Thomas J, Chandler J, Cumpston M, Li T, Page MJ, et al. Cochrane handbook for systematic reviews of interventions. John Wiley \& Sons; 2019.

26. Deeks JJ, Higgins JP, Altman DG. Analysing data and undertaking meta-analyses. Cochrane handbook for systematic reviews of interventions. 2019:241-84.

27. Michie S, Wood CE, Johnston M, Abraham C, Francis JJ, Hardeman W. Training to code intervention descriptions using Behaviour Change Technique Taxonomy version 1 (study 3). Behaviour change techniques: the development and evaluation of a taxonomic method for reporting and describing behaviour change interventions (a suite of five studies involving consensus methods. randomised controlled trials: NIHR Journals Library; 2015.

28. Valentine JC, Pigott TD, Rothstein HR. How many studies do you need? A primer on statistical power for meta-analysis. Journal of Educational Behavioral Statistics. 2010;35(2):215-47.

\section{Supplementary Files}


This is a list of supplementary files associated with this preprint. Click to download.

- Additionalfile1.docx 